\title{
Influence d'un courant sur la réflexion de la houle par une plaque immergée
}

\author{
V. Rey ${ }^{1}$, R. Capobianco 2 , C. Dulou ${ }^{3}$ \\ 1 Mâ̂tre de Conférences, LSEETEISITV, Université Toulon-Var, \\ BP 132, 83957 La Garde, E-mail : vincent.rey@lseet.univ-tln.fr, \\ Tel : 0494142464 \\ ${ }^{2}$ Doctorant, LEPIEISITV, Université de Toulon-Var, BP 56, 83162 \\ La Valettedu Var, E-mail : capobian@isitv.univ-tln.fr, Tel :0494 \\ 142351 \\ 3 ATER, DGO, Université Bordeaux I, Av. des Facultés, 33405 \\ Talence, E-mail : c.dulou@epoc.u-bordeaux.fr, Tel : 05568488 \\ 53
}

Résumé :

Le but de ce travail expérimental était d'étudier, pour une houle d'incidence normale, l'influence d'un courant homogène dans le sens de propagation de la houle sur le pouvoir de réflexion d'une plaque immergée. Les résultats expérimentaux de réflexion en fonction de la période de la houle (dans un repère fixe) et pour différentes amplitudes ont montré une faible influence du courant d'une part sur les positions des maxima et des minima de réflexion de la houle, et d'autre part sur leur intensité. Les positions des maxima d'énergie réfléchie sont donc peu affectées tandis que l'énergie réfléchie est moindre en présence d'un courant. En outre, les réflexions mesurées pour différentes amplitudes d'ondes de fréquence donnée, ou pour des ondes bichromatiques montrent un comportement des houles correctement décrit par une approche linéaire.

Abstract :

The purpose of this experimental work was the study of the influence of an homogeneous current in the incident wave direction on the wave reflecting power of a submerged plate. Results concerning reflection versus the wave period (in a fixed frame) for various amplitudes have shown a weak influence of the current both on the maxima and minima locations and on their amplitude. The locations of the maximum reflected energy are then weakly modified by the current contrary to the energy which is decreased. Moreover, reflections 


\section{VII ${ }^{\text {emes }}$ Journées Nationales Génie Civil - Génie Côtier, Anglet, France, 15-17 Mai 2002}

measured either for various amplitudes at given frequency, or for bichromatic waves, show a wave behaviour correctly described by a linear approach.

\section{Introduction}

En zone côtière, la houle peut être partiellement réfléchie par des variations rapides du fond. Ce "pouvoir de réflexion" des variations du fond a conduit à de nombreuses études et à la réalisation d'ouvrages côtiers immergés destinés à protéger des aménagements ou sites naturels de l'impact de la houle par réflexion partielle de son énergie (voir par exemple Rey, 1992; Rey, 1995).

Des ouvrages de type plaque horizontale immergée ont été proposés pour la protection côtière. Des études linéaires de leur pouvoir de réflexion en fonction de la longueur d'onde des vagues ont montré la présence de maxima et minima de réflexion (Patarapanich, 1984; Sturova, 1991). Ce caractère oscillant de l'intensité de l'énergie réfléchie est dû à un phénomène d'interférence au-dessus de la plaque. Les aspects non-linéaires ont fait l'objet d'études plus récentes (voir Brossard et Chagdali, 2001).

En zone côtière, des courants de l'ordre de 10 à $100 \mathrm{~cm} / \mathrm{s}$ sont souvent observés, induits par la marée ou par le déferlement des houles. Ils ont un effet parfois important sur la vitesse de propagation et la direction de la houle (Phillips, 1977), et par conséquent doivent a priori avoir une influence sur l'efficacité des brise-lames.

L'objet de ce travail, réalisé dans le Bassin de Génie Océanique (BGO) FIRST, La Seyne-sur-Mer, France, est l'étude de la réflexion de la houle par une plaque immergée, pour des houles monochromatiques ou bichromatiques superposées à des courants homogènes et de même direction que la houle, modifiant par conséquent les caractéristiques de sa propagation. Le disposif expérimental et la méthode de calcul du coefficient de réflexion sont présentés dans le deuxième paragraphe. Les résultats concernant le coefficient de réflexion en fonction de la période de la houle sont présentés et discutés dans le troisième paragraphe.

\section{Expériences et méthodes de mesure}

\subsection{Dispositif expérimental}

Le BGO First a une longueur utile de $24 m$, une largeur utile de $16 \mathrm{~m}$; sa profondeur maximale utile est de $5 \mathrm{~m}$ et la profondeur de son puits central $10 \mathrm{~m}$. Il est équipé d'un générateur de courant, d'un générateur de houle sur toute la largeur et d'un chariot XY mobile selon deux axes perpendiculaires. 


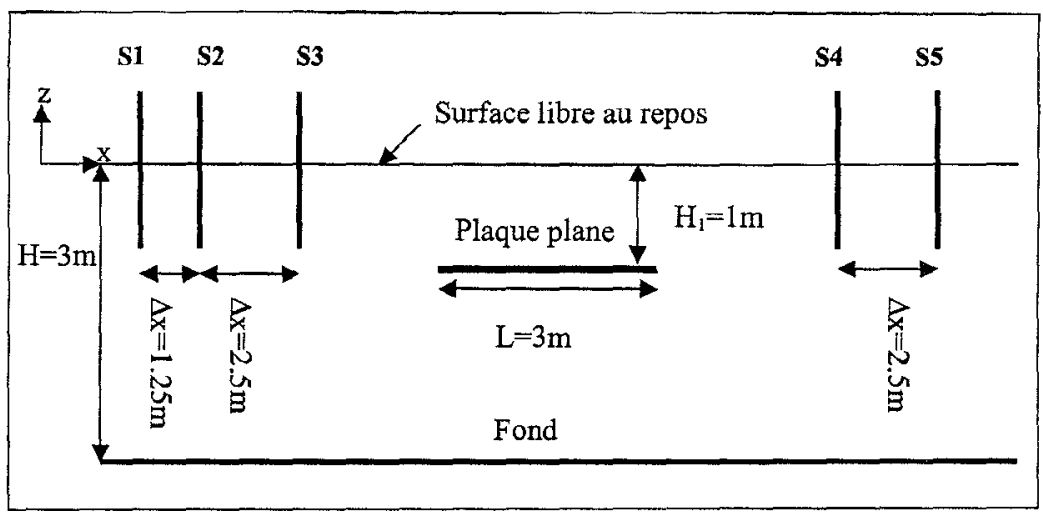

FIG. 1 - Dispositif expérimental - Experimental set-up

A l'autre extrémité, un amortisseur de houle de forme parabolique est perforé pour permettre les études avec superposition d'un courant. Un fond mobile permet d'ajuster la profondeur d'eau à la valeur désirée et d'intervenir rapidement sur l'instrumentation et les maquettes fixées sur le fond. Un puits central de 5 mètres de diamètre permet d'étudier des structures par grande profondeur. Le fond du bassin peut également être incliné de $\pm 7 \%$ pour les applications de génie côtier par profondeur variable. Pour l'étude réalisée, une paroi a été fixée dans la longueur du bassin, réduisant sa largeur à $2 \mathrm{~m}$. Cette paroi était constituée de la juxtaposition de plaques de contreplaqué marine, d'épaisseur $3 \mathrm{~cm}$ et de largeur $1.54 \mathrm{~m}$; elles étaient maintenues par des fixations au dessus de la surface libre. La profondeur d'eau était constante et égale à $3 m$. La plaque horizontale étudiée, en contreplaqué marine de longueur $3 m$, et d'épaisseur $3 \mathrm{~cm}$, était immobile et fixée à $1 \mathrm{~m}$ au dessous de la surface libre au repos (voir Fig. 1).

Le générateur de houle, composé de flotteurs oscillant horizontalement, permet de générer des houles régulières ou irrégulières, dans la direction longitudinale, de hauteur crête à creux maximale $0.8 \mathrm{~m}$ pour des périodes comprises entre 0.7 et $3.4 s$. Les houles générées dans le cadre de cette étude étaient régulières, de périodes comprises entre 1.3 et $3.5 \mathrm{~s}$, et d'amplitudes comprises entre 0.025 et $0.2 \mathrm{~m}$ selon les périodes. La génération d'un courant constant et homogène s'effectue grâce à l'action de pompes pilotées à partir de mesures de vitesse de l'eau. Nos études ont été réalisées pour des courants $U$ d'intensités $0,0.05,0.1$ et $0.2 \mathrm{~m} / \mathrm{s}$ dans le sens de propagation de la houle.

Les mesures de la houle étaient effectuées grâce à cinq sondes capacitives synchrones, notées $S_{1}$ à $S_{5}$. Trois sondes en amont de la structure et deux en aval permettaient de mesurer ondes réfléchie et transmise. Leurs espacements 
étaient tels que $x_{3}-x_{2}=x_{5}-x_{4}=2\left(x_{2}-x_{1}\right)=2.5 \mathrm{~m}$ où $x_{i}, i=1, . ., 5$ sont les positions respectives des sondes $S_{i}$. Afin que la mesure de l'onde réfléchie ne soit pas perturbée par les modes locaux (non propagatifs), les sondes $S_{1}$ à $S_{3}$ étaient positionnées suffisamment loin de la structure (6m côté amont pour $\left.S_{3}\right)$.

La fréquence d'acquisition était de $16 \mathrm{~Hz}$, la durée de chaque mesure environ 2 min. Le traitement de Fourier des signaux calibrés permettait d'obtenir au niveau de chacune des sondes l'amplitude, la fréquence et la phase de chaque composante du signal.

\subsection{Mesures de la réflexion de la houle}

Le coefficient de réflexion $R$ de la houle était mesuré avec une méthode à trois sondes basée sur les moindres carrés. Cette méthode, qui est basée sur celle proposée par Mansard et Funke (1980) et appliquée ici au cas de houles superposées à un courant de même sens, nécessite la connaissance de l'amplitude et de la phase de l'onde au niveau de chacune des sondes.

Elle est basée sur l'hypothèse d'une houle linéaire. Loin de la structure, la déformée de la surface libre $\eta(x, t)$ pour une onde de fréquence $f=\omega / 2 \pi$ est la résultante de deux ondes planes se propageant suivant la direction $O x$ dans les sens opposés :

$$
\eta(x, t)=\left\{a^{-} e^{-i k^{-} x}+a^{+} e^{+i k^{+} x}\right\} e^{i \omega t}
$$

avec $a^{-}$et $a^{+}$amplitudes complexes. $k^{\mp}$ sont les nombres d'onde des ondes propagatives incidente et retrograde, ils sont donnés par

$$
\left(\omega \pm U k^{ \pm}\right)^{2}=\sigma^{ \pm}=g k^{ \pm} \tanh \left(k^{ \pm} h\right)
$$

où $\sigma^{ \pm}$sont les fréquences relatives dans un repère en translation à la vitesse $U$. Les vitesses de phase $C^{ \pm}$et de groupe $C_{g}^{ \pm}$définies dans le repère en translation sont données par

$$
\begin{aligned}
& C^{ \pm}=\frac{\sigma^{ \pm}}{k^{ \pm}} \\
& C_{g}^{ \pm}=\frac{1}{2} C^{ \pm}\left[1+\frac{2 k^{ \pm} h}{\sinh 2 k^{ \pm} h}\right]
\end{aligned}
$$

L'expression "théorique" de la déformée de la surface libre au niveau de la sonde $S_{n}$, basée sur une houle d'Airy (1) est donnée, après un choix arbitraire de l'origine des temps, par :

$$
\eta_{n}=\left\{a_{i} e^{-i\left(k^{-} x_{1}+\Delta n^{-}\right)}+a_{r} e^{+i\left(k^{+} x_{1}+\Delta n^{+}+\varphi\right)}\right\} e^{i \omega t}
$$


avec $\Delta_{n}^{-}=k^{-}\left(x_{n}-x_{1}\right)$ et $\Delta_{n}^{+}=k^{+}\left(x_{n}-x_{1}\right)$, $a_{i}$ et $a_{r}$ les amplitudes de l'onde incidente et de l'onde réfléchie, et $\varphi$ le déphasage de l'onde réfléchie. La composante fondamentale de la déformée de la surface libre mesurée par la sonde $S_{n}(n=1,2,3)$ est de la forme :

$$
\eta_{n}^{(m)}=A_{n} e^{i\left(\omega t-\varphi_{n}\right)}
$$

où l'exposant $(m)$ signifie valeur mesurée. Les amplitude et phase $A_{n}$ et $\varphi_{n}$ sont calculées par analyse de Fourier du signal mesuré par la sonde $S_{n}$. Les amplitudes $a_{i}$ et $a_{r}$, et par conséquent le coefficient de réflexion $R=\left|\frac{a_{r}}{a_{i}}\right|$ peuvent être calculés à partir des données des sondes $S_{1}$ à $S_{3}$ par une méthode des moindres carrés, les mesures des sondes étant synchronisées. Les expressions (5) et (6) sont réécrites sous les formes respectives

$$
\begin{aligned}
\eta_{n} & =\left\{b_{i} e^{-i \Delta_{n}^{-}}+b_{r} e^{+i \Delta_{n}^{+}}\right\} e^{i \omega t} \\
\eta_{n}^{(m)} & =B_{n} e^{i \omega t}
\end{aligned}
$$

avec $b_{i}=e^{-i k^{-} x_{1}}, b_{r}=e^{+i\left(k^{+} x_{1}+\varphi\right)}, B_{n}=A_{n} e^{-i \varphi_{n}}$. On minimise l'erreur $E^{2}$ définie par :

$$
E^{2}=\sum_{n=1}^{3}\left[b_{i} e^{-i \Delta_{n}^{-}}+b_{r} e^{+i \Delta_{n}^{+}}-B_{n}\right]^{2}
$$

en écrivant $\frac{\partial E^{2}}{\partial b_{i}}=\frac{\partial E^{2}}{\partial b_{r}}=0$. On en déduit les modules des amplitudes des ondes incidente et réfléchie $a_{i}$ et $a_{r}$

$$
\begin{aligned}
& \left|a_{i}\right|=\left|b_{i}\right|=\left|\frac{s_{2} s_{3}-s_{12} s_{4}}{s_{5}}\right| \\
& \left|a_{r}\right|=\left|b_{r}\right|=\left|\frac{s_{1} s_{4}-s_{12} s_{3}}{s_{5}}\right|
\end{aligned}
$$

avec

$$
\begin{aligned}
& s_{1}=\sum_{n=1}^{3} e^{-2 i \Delta_{n}^{-}} ; \quad s_{2}=\sum_{n=1}^{3} e^{+2 i \Delta_{n}^{+}} ; \quad s_{12}=\sum_{n=1}^{3} e^{+i\left(\Delta_{n}^{+}-\Delta_{n}^{-}\right)} \\
& s_{3}=\sum_{n=1}^{3} A_{n} e^{-i\left(\Delta_{n}^{-}+\delta_{n}\right)} ; \quad s_{4}=\sum_{n=1}^{3} A_{n} e^{+i\left(\Delta_{n}^{+}-\delta_{n}\right)} ; \quad s_{5}=s_{1} s_{2}-s_{12}^{2}
\end{aligned}
$$

Le coefficient de réflexion du mode fondamental de l'onde est alors donné $\operatorname{par} R=\frac{\left|b_{r}\right|}{\left|b_{i}\right|}$. 


\section{VII ${ }^{i m e s}$ Journées Nationales Génie Civil - Génie Côtier, Anglet, France, 15-17 Mai 2002}

\section{Résultats et interprétations}

\subsection{Coefficient de réflexion des ondes}

On a représenté sur la Fig. 2 le coefficient de réflexion d'une onde monochromatique induit par la plaque plane, et sur la Fig. 3 celui de chacune des deux composantes d'une onde bichromatique. Les ondes bichromatiques étudiées étaient la superposition de houles de périodes $T_{1}$ et $T_{2}$ telles que $\left(T_{1}, T_{2}\right)=(2.59 \mathrm{~s}, 1.79 \mathrm{~s})$ ou $(2.59 \mathrm{~s}, 1.28 \mathrm{~s})$. Le calcul théorique en l'absence de courant $(U=0)$ est basé sur une formulation intégrale des conditions de continuité de vitesse et de pression aux abscisses $x=0$ et $x=L$, il suppose un écoulement potentiel, et des ondes de faible amplitude. Il fait apparaître des maxima et minima de réflexion, qui traduisent un phénomène d'interférence de l'onde au dessus de la plaque, phénomène déjà largement étudié pour la réflexion des ondes de gravité en milieu côtier par des modulations du fond ou des structures immergées (voir par exemple Rey, 1992; Rey, 1995; Rey et al, 1996, Brossard et Chagdali, 2001 et les articles qui y sont cités).

Pour les houles de plus basses fréquences, les amplitudes expérimentales étaient choisies assez faibles pour éviter des efforts de pression trop importants sur les structures et parois à l'origine de rupture de la plaque immmergée et de la paroi latérale. Les réflexions mesurées pour des ondes de fréquence donnée restent faiblement dépendantes des amplitudes des ondes pour la gamme d'amplitudes testées, et celles de chacune des deux composantes des ondes bichromatiques semblables à celles mesurées dans le cas monochromatique.

Les résultats restent assez homogènes aux faibles périodes (inférieures à $T=2 s$ ). Bien que bruités, ils présentent par ailleurs des disparités assez importantes au-delà, dans une gamme de période où la réflexion prévue par la théorie (en l'absence de courant) devient importante. Ces bruits et dispersions des résultats peuvent s'expliquer pour partie par les conditions d'expérience et pour partie par la méthode de calcul du coefficient de réflexion.

D'un point de vue expérimental on a pu observer, lorsque la réflexion est assez importante, une mauvaise tenue de la séparation latérale installée qui avait tendance à osciller assez fortement (amplitude de l'ordre de $5 \mathrm{~cm}$ ) dans la direction transversale. Ceci s'explique d'un point de vue des efforts par une différence de pression importante de part et d'autre de ces plaques latérales, due à la présence d'une onde partiellement stationnaire du côté de l'expérience à cause de la plaque partiellement réfléchissante, et d'une onde progressive de l'autre côté. On observe pour que la réflexion mesurée est en moyenne la plus importante au dessus de $T=2 s$, ce qui est prévu théoriquement, mais que la disparité des résultats en fonction de la période est très importante dans cette gamme de périodes. 


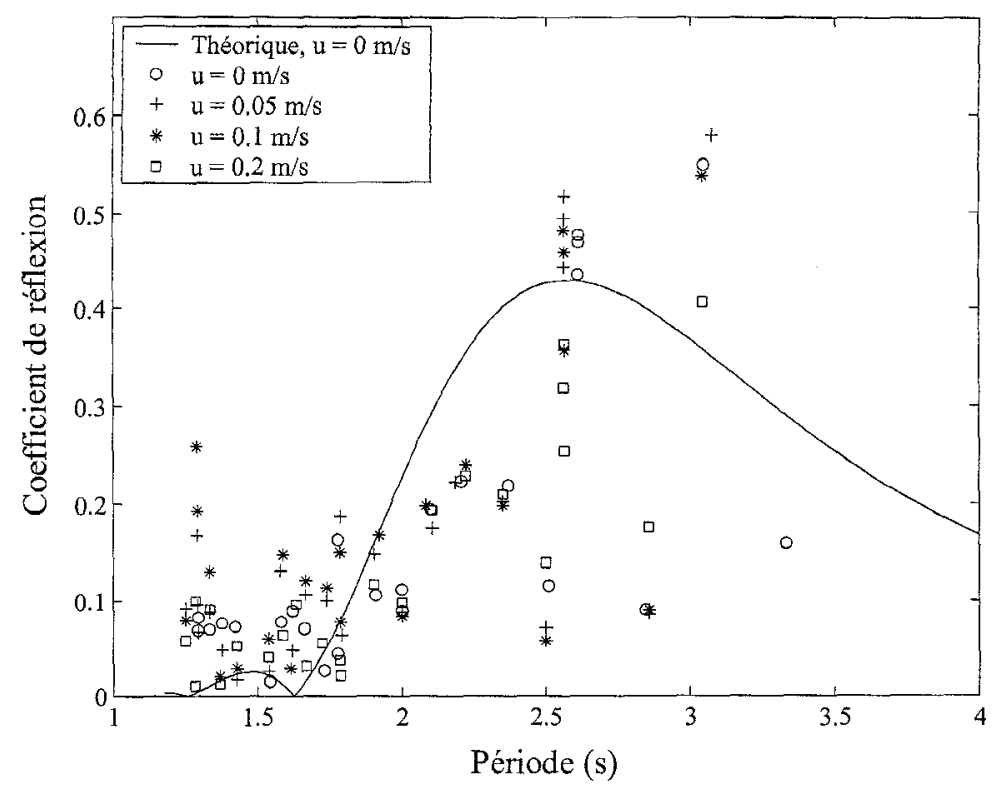

FIG. 2 - Coefficient de réflexion en fonction de la période (ondes monochromatiques)- Reflection coefficient versus wave period (monochromatic waves)

D'un point de vue du traitement des données, un calcul à trois sondes a été choisi afin de minimiser les erreurs, qui proviennent d'une part des incertitudes expérimentales (courant supposé homogène et de valeur donnée, position des capteurs) et des approximations "théoriques" du signal de houle (notamment hypothèse linéaire), et d'autre part de la méthode elle-même. Les erreurs sur le coefficient de réflexion seront d'autant plus importantes qu'on sera proche des conditions pour lesquelles la méthode n'est pas valable, c'est à dire lorsque le coefficient $s_{5}$, défini dans (13) devient nul. L'expression $s_{5}$ peut être réécrite sous la forme

$-\frac{s_{5}}{4}=\left[\frac{e^{i \Delta_{3}^{+}}-e^{-i \Delta_{3}^{-}}}{2 i}\right]^{2}+\left[\frac{e^{i \mu \Delta_{3}^{+}}-e^{-i \mu \Delta_{3}^{-}}}{2 i}\right]^{2}+\left[\frac{\left.e^{i\left(\mu \Delta_{3}^{+}-\Delta_{3}^{-}\right)}-e^{-i\left(\mu \Delta_{3}^{-}-\Delta_{3}^{+}\right.}\right)}{2 i}\right]^{2}$

avec $\mu=\Delta_{2} / \Delta_{3}$. Expression qui s'annule si $e^{-i\left(\Delta_{3}^{-}-\Delta_{3}^{+}\right)}=e^{-i \mu\left(\Delta_{3}^{-}-\Delta_{3}^{+}\right)}=1$ c'est-à-dire si $\left(\Delta_{3}^{-}+\Delta_{3}^{+}\right)=6 \pi, 12 \pi$, etc....

Dans le cadre de notre étude expérimentale, $\mu=2 / 3$, et les valeurs de la période $T$ correspondantes dans la gamme d'étude sont données dans la Table ci-dessous.

\begin{tabular}{|c|c|c|c|c|}
\hline & $U=0 \mathrm{~m} / \mathrm{s}$ & $U=0.05 \mathrm{~m} / \mathrm{s}$ & $U=0.1 \mathrm{~m} / \mathrm{s}$ & $U=0.2 \mathrm{~m} / \mathrm{s}$ \\
\hline$T(\mathrm{~s})$ & 1.2654 & 1.2674 & 1.2735 & 1.3085 \\
\hline$\left(\triangle_{3}^{-}+\Delta_{3}^{+}\right)$ & $6 \pi$ & $6 \pi$ & $6 \pi$ & $6 \pi$ \\
\hline
\end{tabular}




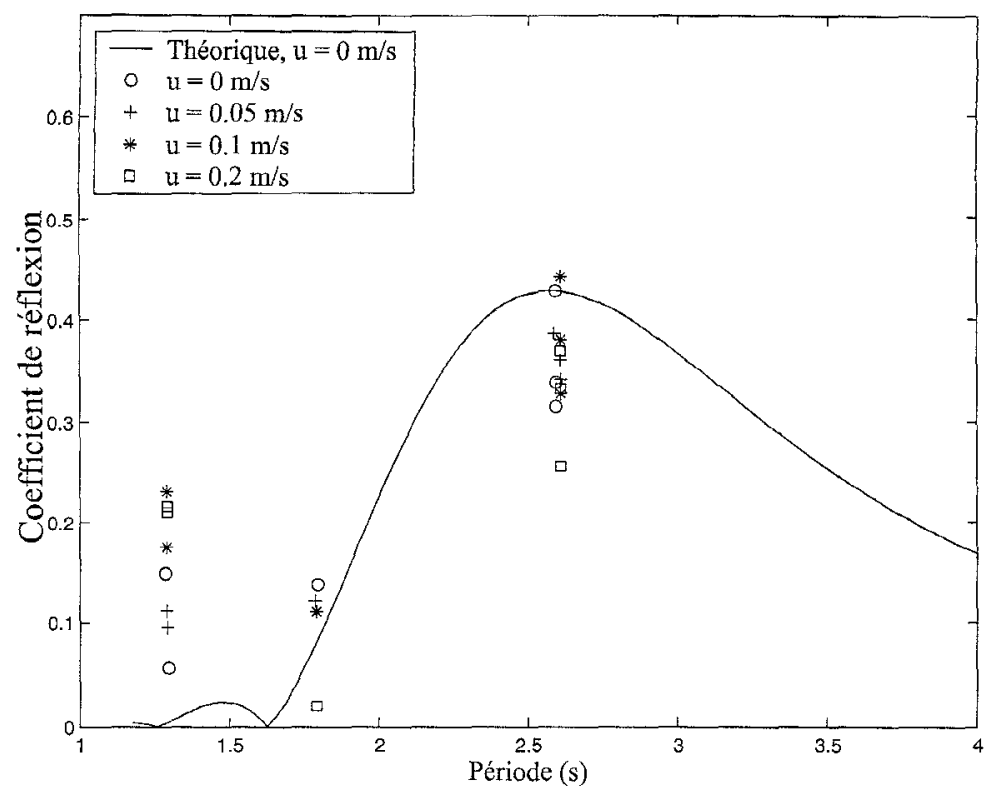

FIG. 3 - Coefficient de réflexion en fonction de la période (onde bichromatique)Reflection coefficient versus wave period (bichromatic wave)

Table 1 : Période (et déphasage correspondant) pour laquelle $s_{5}=0$.

En l'absence de courant, l'expression (14) se réduit à $\sin ^{2}\left(\Delta_{3}\right)+\sin ^{2}\left(\mu \Delta_{3}\right)+$ $\sin ^{2}\left(\Delta_{3}-\mu \Delta_{3}\right)=0$, expression trouvée par Isaacson (1991) et qui s'annule pour $\Delta_{3}=3 \pi, 6 \pi$, etc...

\subsection{Influence du courant sur l'énergie réfléchie}

La courbe théorique, qui tient compte des modes évanescents, des Figs. 2 et 3 montre la présence de maxima et de minima (réflexion nulle), que l'on peut expliquer par un phénomène d'interférence au-dessus de la plaque. En effet, au niveau des discontinuités du milieu (bord d'attaque, $x=0$, et bord de fuite, $x=L$ ), l'onde est partiellement réfléchie, ce qui induit des "aller-retour" de l'onde au dessus de la plaque. L'onde aura tendance à être fortement réfléchie lorsqu'en $x=0$, l'interférence sera constructive c'est-à-dire que les ondes incidente et réfléchie (par le bord de fuite) seront en phase; elle sera complètement transmise dans le cas contraire. Nous avons représenté sur la Fig. 4 la phase relative de l'onde réfléchie après un aller-retour au-dessus de la plaque dans l'approximation d'une onde plane, c'est-à-dire en ne pas considérant les déphasages supplémentaires induits par les modes locaux. On constate qu'en l'absence de courant, la réflexion est maximale pour $T \simeq 1.4 \mathrm{~s}$ et $T \simeq 2.3 \mathrm{~s}$, et minimale pour $T \simeq 1.3 \mathrm{~s}$ et $T \simeq 1.7 \mathrm{~s}$. Ces résultats sont en bon accord avec les extremums observés sur la Fig. 1 pour le calcul théorique. 


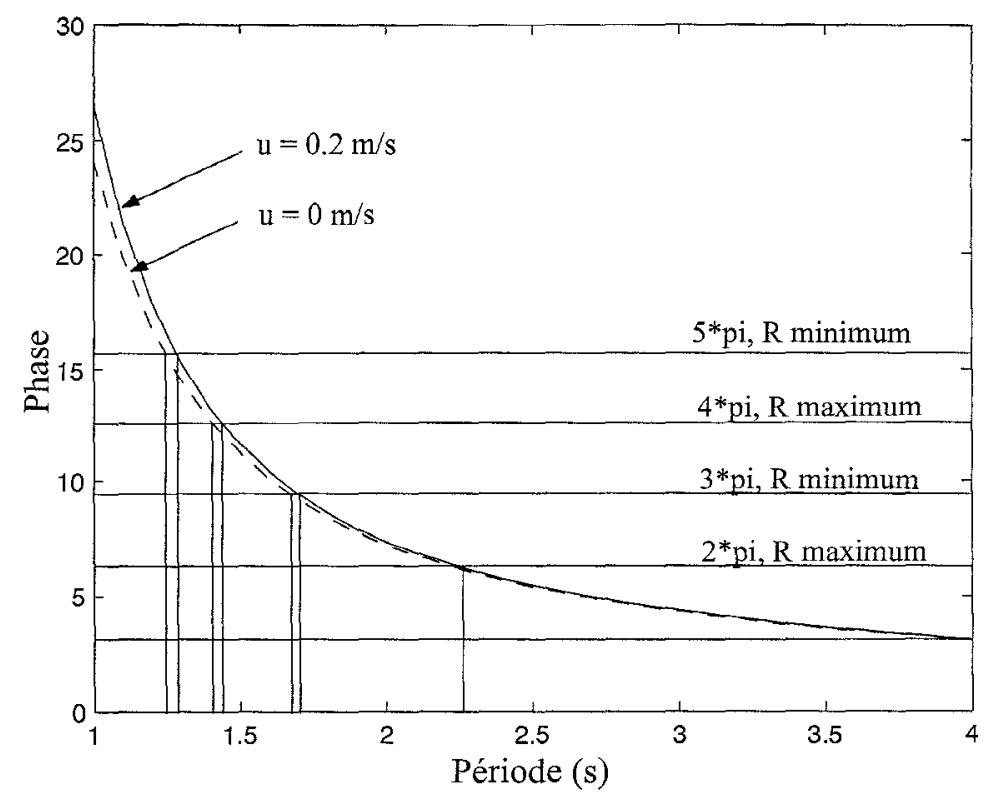

FIG. 4 - Phase de l'onde après un "aller-retour" au dessus de la plaque - Phase lag after a return path above the plate

Les données expérimentales sont bruitées à cause des incertitudes énoncées dans le paragraphe précédent. Cependant, on observe sur les Figs. 2 et 3 que les coefficients de réflexion mesurés en l'absence de courant et pour des courants $U$ non nuls sont similaires, en intensité et d'un point de vue de la position des extremums.

En ce qui concerne les positions des extremums, elles sont en bon accord avec les calculs de phase présentés Fig. 4, qui montraient une faible influence du courant sur l'évolution de la phase après un "aller-retour" de l'onde audessus de la plaque. Cette faible différence de phase, malgré l'écart important entre les nombres d'onde $k^{-}$et $k^{+}$, notamment aux faibles périodes, s'explique par une compensation des effets Doppler dans les directions incidente et rétrograde. Ce résultat montre qu'une structure conçue pour réfléchir un maximum d'énergie pour une gamme de fréquence donnée, restera efficace en présence d'un courant, puisque les périodes de réflexion maximales (ou minimales) sont très peu dépendantes de l'intensité du courant.

En ce qui concerne l'amplitude, on observe qu'elle n'est pas significativement affectée par le courant (pas de tendance à l'augmentation ou à la diminution en comparaison des incertitudes). Cependant, l'intérêt de la structure est sa capacité à réfléchir une partie de l'énergie, qui n'est plus simplement donnée par le rapport du carré des amplitudes réfléchie et incidente, mais par une expression faisant apparaitre un paramètre "d'effet Doppler". La conservation du flux d'énergie moyenne $E_{t}$ de la houle à travers le plan $y 0 z$, normal à sa 


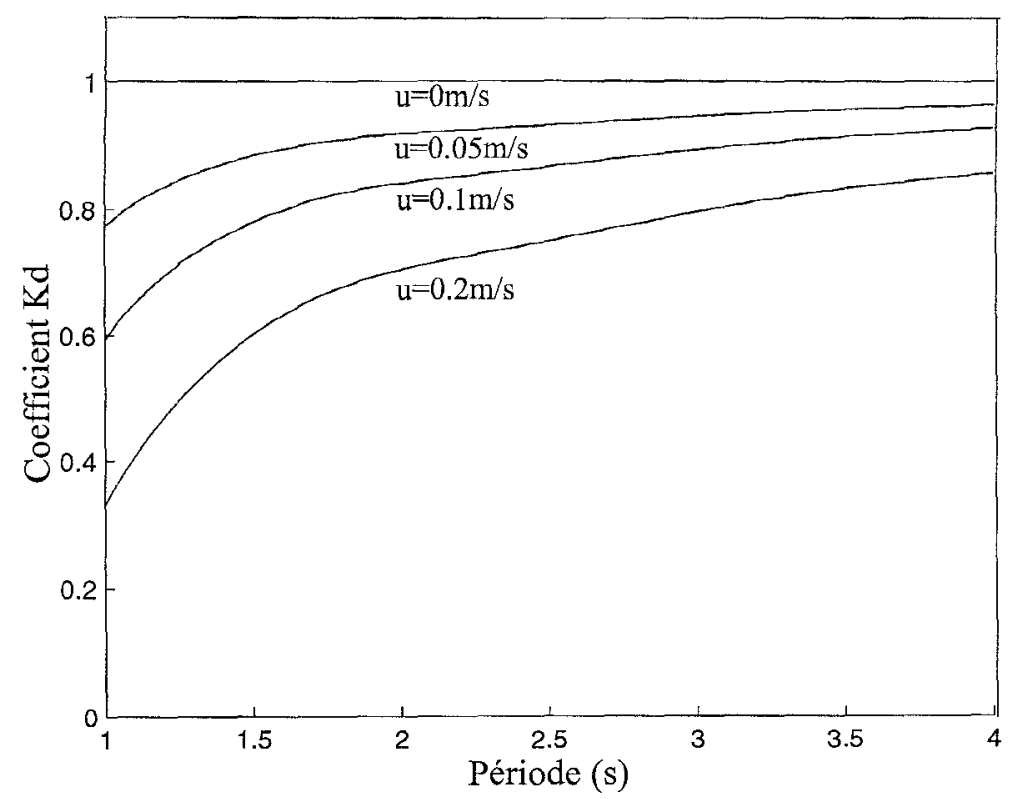

Fig. 5 - Coefficient $K_{d}$ en fonction de la période - Coefficient $K_{d}$ versus wave period

direction de propagation est donné dans le repère en translation à la vitesse $U$ par :

$$
\frac{\partial E_{t}}{\partial x}=\frac{\partial}{\partial x}\left[\frac{E\left(U+C_{g}^{-}\right)}{\sigma^{-}}\right]=\frac{\partial}{\partial x}\left[\frac{E\left(-U+C_{g}^{+}\right)}{\sigma^{+}}\right]=0
$$

Le taux $\tau=\frac{E_{t}^{r}}{E_{t}^{t}}$ d'énergie réfléchie est donné par

$$
\tau=\frac{a_{r}^{2}}{a_{i}^{2}} \frac{\sigma^{-}}{\sigma^{+}} \frac{\left(C_{g}^{+}-U\right)}{\left(C_{g}^{-}+U\right)}
$$

qu'on peut écrire sous la forme $\tau=\frac{a_{r}^{2}}{a_{i}^{2}} K_{d}$, où $K_{d}$ est un coefficient de "Doppler". Ce coefficient est présenté en fonction de la période pour les différentes valeurs expérimentales du courant sur la Fig. 5 . Il fait apparaître une énergie réfléchie d'autant plus faible que le courant est important, pour un rapport donné entre les amplitudes réfléchie et incidente.

\section{Discussion et conclusion}

Les expériences présentées et analysés dans ce travail ont été menées dans un bassin d'essai qui présentait l'avantage de générer des houles superposées à des courants. Ce travail a permis de montrer qu'en présence d'un courant superposé à une houle incidente, l'energie réfléchie par une structure immergée 
de type plaque plane est inférieure à celle mesurée en l'absence de courant, mais que sa plage d'efficacité reste inchangée. L'étude du processus d'interférence au-dessus de la plaque, basé sur la théorie linéaire des ondes, supposées planes, nous donne une information quantitative des maxima et minima de réflexion, comme déjà observé par Patarapanich (1984) en l'absence de courant. Le poids des modes locaux sur l'évolution de la phase de l'onde au voisinage des discontinuités reste donc très inférieur à celui observé pour des obstacles rectangulaires immergés (Rey et al, 1992), où l'étude basée sur l'approximation onde plane ne permettait qu'une estimation qualitative de la position des extremums. En outre, les résultats pour différentes amplitudes à fréquence donnée ou pour la superposition de deux fréquences à amplitudes données montrent une faible influence de l'amplitude sur le coefficient de réflexion, ce qui d'une part conforte les études théoriques basées sur des houles linéaires (ou composées de la superposition linéaire de houles linéaires) et d'autre part nous permet de conforter l'hypothèse linéaire sur laquelle est basée la méthode d'analyse des signaux de houle mesurés au niveau des sondes.

En ce qui concerne l'expérience, nous avions observé le problème de mouvement transversal de la "fausse" paroi latérale du bassin, qui a considérablement perturbé l'onde réfléchie par la plaque lorsque cette réflexion était importante. Cependant, les résultats sont restés suffisamment consistants pour une analyse qualitative de la réflexion de la houle par la plaque immergée. Quant à la méthode de mesure, elle a montré qu'elle était beaucoup plus précise que la méthode à deux sondes (non présentée ici, voir par exemple Goda et Suzuki., 1976), pour laquelle de fortes disparités entre résultats (sondes $S_{1}$ à $S_{3}$ utilisées 2 à 2) pouvaient être observées, notamment lorsqu'au moins une des trois distances $x_{j}-x_{i}$ était proche des conditions de divergence de la méthode $\left(\left(k^{-}+k^{+}\right)\left(x_{j}-x_{i}\right)=2 n \pi, n\right.$ entier $)$.

\section{Remerciements :}

Les auteurs remercient le Conseil Général du Var pour son aide financière qui a permis la réalisation des expériences dans le BGO First.

\section{Références :}

Brossard, J. and Chagdali, M., 2001 : Experimental investigation of the harmonic generation by waves over a submerged plate. Coastal Engineering., 42, 277-290.

Goda, Y. and Suzuki, Y., 1976 : Estimation of incident and reflected waves in 
random wave experiments. Proc. 15th Coastal Engrg. Conf., 1, 828-845.

Isaacson, M., 1991 : Measurement of regular wave reflection. Journal of Waterway, Port, Coastal and Ocean Engineering., 117 (6), 553-569.

Mansard, E. P. D. and Funke, E. R., 1980 : The measurement of incident and reflected spectra using a least square method. Proc. 17th Coastal Engrg. Conf., 1, 154-172.

Patarapanich, M., 1984 : Maximum and zero reflection from submerged plate. Journal of Waterway, Port, Coastal and Ocean Engineering., 110 (2), 171-181. Phillips, O. M., 1977, The Dynamics of the Upper Ocean (Cambridge University Press, 2nd edition, 1977).

Rey, V., 1992 : Propagation and local behaviour of normally incident gravity waves over varying topography, European Journal of Mechanics B/Fluids, 11, 213-232.

Rey, V., 1995 : A note on the scattering of obliquely incident surface gravity waves by cylindrical obstacles in waters of finite depth, European Journal of Mechanics B/Fluids, 14 (1), 207-216.

Rey, V., Belzons, M. and Guazzelli, E. 1992 Propagation of surface gravity waves over a rectangular submerged bar, J. Fluid Mech., 235, 453-479.

Rey, V., Guazzelli, E. and Mei, C. C. 1996 : Resonant reflection of surface gravity waves by one-dimensional doubly sinusoidal beds, Physics of Fluids, 8 (6), 1525-1530.

Sturova, V., 1991 : Propagation of plane surface waves over an underwater obstacle and a submerged plate, Journal of applied mechanics and technical physics, 32 (3), 453-479. 